\title{
Seguridad Internacional International Security
}

\author{
Thomas Gil \\ Technische Universität Berlin \\ thomas.gil@tu-berlin.de
}

\author{
Recibido / received: 13/03/2018 \\ Aceptado / accepted: 25/06/2018 \\ DOI: https://doi.org/10.20318/eunomia.2018.4351
}

\section{Resumen}

La seguridad internacional es algo más que la ausencia de inseguridad e inestabilidad en las relaciones entre agentes sociales tanto nacionales como internacionales. Como "bien relacional" presupone una serie de condiciones para poder darse.

\section{Palabras clave}

Seguridad, concepciones de la seguridad, seguridad nacional, seguridad militar, seguridad económica, desarrollo humano.

\begin{abstract}
Originally, international security was conceived in a restrictive sense as national, state, or military security. Different circumstances, events, and the theorizing about them, however, have led to broaden and to deepen the conception of it.
\end{abstract}

\section{Keywords}

Security, security conception, national security, militar security, economic security, human development.

\footnotetext{
SUMARIO. 1. Introducción. 2. Ampliación del concepto de seguridad internacional. 3. Seguridad internacional como cualidad de las relaciones internacionales. 4 . ¿Cómo cabe conseguirla?
}

\section{Introducción}

La historia de los estudios sobre seguridad internacional -de los que Browning (2013) nos ofrece una útil visión panorámica- nos revela que esta noción tiene muchas más facetas y dimensiones de las que cabría suponer a primera vista. Por de pronto, convendría delimitar su ámbito, distinguiendo la seguridad internacional de otros tipos de seguridad, cuál sería el caso de la seguridad social, la seguridad ciudadana, la seguridad informática, la seguridad jurídica, la seguridad laboral, la seguridad bancaria, la seguridad vial, etc. 
La seguridad internacional abarca muchos y muy diversos aspectos específicos que a lo largo de la historia de los estudios sobre esta materia se han ido poniendo de relieve, al irse convirtiendo en el centro de la atención de los respectivos análisis. Así las cosas, en los años de lo que se dio en llamar "guerra fría", la seguridad internacional significaba ante todo seguridad nacional, siendo esto algo que se ve muy bien reflejado en los oportunos estudios de la época, dedicados mayoritariamente a las estrategias militares de los Estados Unidos y de la Unión Soviética. En ese contexto, dichos estudios se limitaban al uso de las armas y al papel que debía jugar la fuerza militar en un marco internacional conflictivo, donde primaba la rivalidad internacional de las dos superpotencias mundiales.

La proliferación del armamento nuclear, junto a los peligros y riesgos que los arsenales nucleares conllevaban, pusieron de manifiesto la fundamental interdependencia de la idea de seguridad y problematizaron la hasta entonces aceptada equivalencia entre seguridad y fuerza militar.

Una creciente conciencia crítica con respecto a la perspectiva que vinculaba indisolublemente seguridad con potencia militar y satisfacción de intereses primordialmente nacionales propició una diferenciación de la idea de seguridad en el contexto de las relaciones internacionales, que dejó ver la importancia de los distintos sectores dentro de los cuales se articulaba y realizaba la seguridad.

Posteriormente, la perspectiva del desarrollo económico, en particular, y el desarrollo humano, en general, se convirtieron en el centro de atención de los estudios realizados por autores como Barry Buzan (2009), Fisas Armengol (1987), Johann Galtung (2003), J. Jordán (2013), Dieter Senghaas (2012), Amartya Sen y Martha Nussbaum (1997).

\section{Ampliación del concepto de seguridad internacional}

Tal como la paz no significa únicamente ausencia de guerras y conflictos bélicos, sino todo un conjunto de coexistencias y cooperaciones pacíficas, la seguridad internacional es algo más que la ausencia de inseguridad e inestabilidad en las relaciones entre agentes sociales tanto nacionales como internacionales.

A Barry Buzan (2009), entre otros muchos autores, le debemos una diferenciación de los distintos sectores y contextos en los que el tan apreciado bien de la seguridad se podría dar como cualidad específica de las relaciones entre agentes sociales, así como de las condiciones de vida y actuación que éstos tendrían. La distinción propuesta por este autor entre sectores o esferas representa una excelente contribución terminológica a la ampliación del concepto de seguridad y, en general, a una concepción positiva ( $\mathrm{y}$ no simplemente negativa) de la idea de seguridad internacional. Barry Buzan distingue seguridad "militar", seguridad "política", seguridad "económica", seguridad "societal" y seguridad "ecológico-ambiental".

Con arreglo a esta clasificación, la seguridad militar sería el tipo de seguridad en el que se concentraron los análisis tradicionales y abarcaría exclusivamente las capacidades ofensivas y defensivas de los Estados nacionales. Los otros tipos de seguridad rompen con la óptica restrictiva de una exclusiva concentración en la seguridad militar. Así, la seguridad política tendría en cuenta la estabilidad de estructuras institucionales y de organización a disposición de los Estados y sus respectivos gobiernos, mientras que la seguridad económica acentuaría la importancia del acceso real y efectivo por parte de los miembros de una cierta sociedad a recursos y mercados. La seguridad societal, por su parte, incluiría cuestiones de identidad tanto cultural como étnica, acentuando la individualidad de 
las trayectorias biográficas de los agentes individuales y colectivos. Por último, la seguridad ecológico-ambiental se interesaría por cuestiones y condiciones medioambientales, básicas para toda clase de vida individual y colectiva en nuestro planeta.

Es evidente que una concepción ampliada de la seguridad internacional, como la que propone Barry Buzan, acabaría con las restricciones, hoy en día injustificables, de la noción tradicional y exclusivista de la seguridad internacional que, por unilateral e inadecuada, impide el correcto tratamiento de los problemas y retos con los que actualmente nos vemos confrontados.

\section{Seguridad internacional como cualidad de las relaciones internacionales}

La seguridad es un bien relacional que presupone una serie de condiciones para poder darse. Es un bien relacional, no material, aunque lleve, una vez realizado, a diferentes bienes materiales. Es un bien que se da como consecuencia de ciertas actividades y de ciertos condicionamientos sin que se pueda alcanzar de manera directa.

Ciertamente, la seguridad alcanzable de manera directa imponiendo estrategias de fuerza y violencia no es una seguridad que pueda perdurar, no es estable y tiene tasado su tiempo de vigencia. Al ser un bien relacional, más que algo concreto y material, es una cualidad de algo distinto a ella. Vendría a ser como un modo de relacionarse y de entrar en conexión con el mundo social y práctico, con otros agentes y otros sistemas de acción. Expresado en otros términos, se podría decir que la seguridad es siempre la seguridad de algo: de la nación, del Estado, de los individuos, de ciertos grupos étnicos, del medio ambiente, de la economía.

\section{4. ¿Cómo cabe conseguirla?}

Sabiendo qué condiciones propician la realización de la seguridad de los individuos, las instituciones, las relaciones y, en general, de las situaciones y los contextos de las acciones individuales y colectivas, es más fácil indicar en qué consistiría el trabajo de su preparación, realización y fomento. Lo que parece absolutamente claro es que la seguridad internacional no es solamente una cuestión de estrategias de poder, de contención o de disuasión, sino que implica mucho más.

Por otra parte, se requieren toda una serie de cambios y desarrollos que ponen bien de manifiesto la complejidad de los condicionamientos de la seguridad internacional. El cambio climático, con todas sus secuelas, puede trastornar algunos de los equilibrios existentes en la actualidad, toda vez que, por mencionar un solo ejemplo, dicho cambio climático puede conllevar una subida del nivel de los mares con las consiguientes inundaciones de núcleos urbanos costeros y las consiguientes migraciones en masa de las poblaciones que ahora habitan el litoral.

La evolución tecnológica es asimismo fundamental para la configuración de las condiciones concretas de la seguridad internacional. No solamente el desarrollo de la energía nuclear y la proliferación incontrolable de armas atómicas, sino también la fabricación de soldados y pilotos mecánicos (robots), que está haciendo posible el desarrollo de la ciencia robótica, transforman de manera radical las formas tradicionales de acción y hacen necesarios nuevos planteamientos.

Para la seguridad internacional, la seguridad humana resulta fundamental. No sólo Estados e instituciones necesitan el codiciado bien de la seguridad para poder funcionar adecuadamente, sino también lo precisan los individuos para poder 
desarrollar sus facultades humanas. Las personas humanas necesitan, para poder vivir dignamente, condiciones estables y seguras, salarios justos, una educación y una formación profesional adecuadas, cuidados médicos y asistencia social, sin olvidar, por supuesto, un medio ambiente no contaminado. Todos estos bienes y servicios integran esencialmente su seguridad. Sin ellos el desarrollo individual de cada ser humano no es posible. Visto esto desde una perspectiva política y social, se podría afirmar que si los individuos no pueden desarrollarse adecuadamente, se generan asimetrías estructurales y anomías disfuncionales que corroen el convivir colectivo y ponen sistemáticamente en peligro todo orden establecido, afectando a las formas de coexistencia pacíficas que, por muy precarias que sean, constituyen la primera condición imprescindible de toda vida digna y humana.

Bibliografía

BROWNING, C. S. (2013), International Security: A Very Short Introduction, Oxford University Press, Oxford.

BUZAN, B., HANSEN, L. (2009), The Evolution of International Security Studies, Cambridge University Press, Cambridge.

FISAS ARMENGOL, V. (1987), Introducción al estudio de la paz y de los conflictos, Editorial Lerna, Barcelona.

GALTUNG, J. (2003), Paz por medios pacificos: paz y conflicto, desarrollo y civilización, Bakeaz, Bilbao.

JORDÁN, J. (2013), Manual de Estudios Estratégicos y Seguridad Internacional, Plaza y Valdés, Madrid.

NUSSBAUM, M., SEN, A. (1997), The Quality of Life, Clarendon Press, Oxford.

SENGHAAS, D. (2012), Weltordnung in einer zerklüfteten Welt. Hat Frieden Zukunft?, Suhrkamp, Berlin. 